\title{
LILIUM REGALE (LILIACEAE) NATURALIZADA EN LA ARGENTINA Y NUEVOS REGISTROS PARA BRASIL AUSTRAL
}

\author{
Lilium regale (Liliaceae) naturalized in Argentina and new records for southern Brazil
}

\author{
JULIO A. HURRELLO1', GUSTAVO DELUCCHI² \& HÉCTOR A. KELLER ${ }^{3}$
}

\begin{abstract}
Resumen: En este estudio se cita por primera vez a Lilium regale (Liliaceae) como especie naturalizada en Misiones, Argentina, y se suman nuevos registros para el sur de Brasil. Se incluye: descripción de la especie, sinonimia, iconografía, distribución, fenología, polinización, dispersión, nombres vernáculos, usos, materiales de referencia y comentarios sobre su naturalización. Además, se presenta una clave para diferenciar $L$. regale de L. longiflorum, la otra especie de Liliaceae naturalizada en la Argentina.
\end{abstract}

Palabras clave: Argentina, Brasil, bulbosa ornamental, Lilium regale, naturalización.

Summary: In this study Lilium regale (Liliaceae) is cited for the first time for Misiones, Argentina, where it occurs as a naturalized species, and new records for southern Brazil are added. The species description, synonymy, iconography, distribution, phenology, pollination, dispersal, vernacular names, uses, reference materials, and comments about its naturalization are included. A key to differentiate $L$. regale from $L$. longiflorum, the other naturalized Liliaceae in Argentina, is also presented.

Key words: Argentina, Brazil, bulbous ornamental, Lilium regale, naturalization.

\section{Introducción}

En este trabajo se menciona por primera vez a Lilium regale E. $\mathrm{H}$. Wilson (Liliaceae) como naturalizada en la provincia de Misiones, Argentina. Lilium L. comprende 80-110 especies de zonas templadas y áreas montañosas de zonas cálidas del hemisferio norte. Es más abundante en China, donde se hallan 55 especies, de las cuales 35 son endémicas, entre ellas, L. regale (Liang \& Tamura, 2000; Wu et al., 2015). Además, $L$. regale ha sido hallada naturalizada en Brasil, en los estados de Minas Gerais, São Paulo, Paraná, Santa Catarina y Rio Grande do Sul (Schneider, 2007; REFLORA, 2018), y se incluyen aquí dos nuevos registros para Paraná y Santa Catarina, no mencionados con anterioridad.

Tanto $L$. regale como otras especies del género, han sido introducidas en diversos países del mundo por su valor ornamental. El cultivo de especies para esta finalidad constituye una de las principales vías de ingreso de especies exóticas que luego se naturalizan y, eventualmente devienen invasoras o malezas (Reichard \& White, 2001; Bell et al., 2003; Groves et al., 2005; Kowarik, 2005; Birkeland Salvesen, 2006; Dehnen-Schmutz et al., 2007; Foxcroft et al., 2008). En virtud de monitorear el proceso de naturalización de potenciales invasoras, el primer registro de las especies

\footnotetext{
${ }^{1}$ Laboratorio de Etnobotánica y Botánica Aplicada, Facultad de Ciencias Naturales y Museo, Universidad Nacional de La Plata. Calle 64 nro. 3, 1900-La Plata. Investigador CONICET, Argentina. E-mail: juliohurrell@gmail.com

${ }^{2}$ Facultad de Ciencias Naturales y Museo, Universidad Nacional de La Plata. Paseo del Bosque s/nro. 1900-La Plata, Argentina. E-mail: delucchi@fcnym.unlp.edu.ar

${ }^{3}$ Facultad de Ciencias Forestales, Universidad Nacional de Misiones, Instituto de Botánica del Nordeste, Casilla de Correo 209, 3400-Corrientes. Investigador CONICET, Argentina. E-mail: kellerhector@hotmail.com
} 
naturalizadas resulta de especial interés.

Esta contribución incluye la descripción de L. regale, sinonimia, iconografía, distribución, fenología, polinización y dispersión, nombres vernáculos, utilidades, materiales de referencia, una clave para diferenciarla de L. longiflorum, previamente citada como naturalizada en nuestro país (Hurrell et al., 2010), y comentarios sobre su naturalización.

\section{Materiales y Métodos}

A partir de distintos relevamientos florísticos y etnobotánicos desarrollados en la provincia de Misiones, se detectó la presencia espontánea de L. regale en sitios perturbados. Se realizaron distintas observaciones en el terreno y se coleccionó material de herbario de referencia que documenta el hallazgo, depositado en el herbario CTES (Instituto de Botánica del Nordeste, Corrientes). Para la identificación de esta especie se consultó el ejemplar tipo (JSTOR, 2018). Asimismo, se observaron en CTES ejemplares de los estados de Paraná y Santa Catarina, Brasil, que amplían los registros de $L$. regale para ese país, dentro de la continuidad geográfica del Bosque Atlántico (Di Bitetti et al., 2003; Galindo-Leal \& Camara, 2003). Además, se consultaron las bases de datos The Plant List (2013), REFLORA (2018) y Tropicos (2018).

Para los conceptos vinculados a los estudios sobre las invasiones biológicas, en especial, originadas a partir de ornamentales escapadas de cultivo, se consultó la literatura específica: Richardson et al. (2000, 2010, 2011), Reichard $\&$ White (2001), Bell et al. (2003), Groves et al. (2005), Kowarik (2005), Birkeland Salvesen (2006), Pyšek \& Richardson (2006), DehnenSchmutz et al. (2007), Foxcroft et al. (2008), Richardson \& Pyšek (2012) y Randall (2017).

\section{Resultados}

Lilium regale E. H. Wilson, Gard. Chron. ser. 3, 53: 416, fig. 182. 1913.

Typus: China. Sichuan. Western Szechuan: Min Valley, 25-V-1908, Wilson 1446 A (E!).
Lilium myriophyllum E. H. Wilson, Flora \& Sylva 3: 330, pl. 1. 1905 [no Franch., J. Bot. (Morot) 6 (17-18): 313-314. 1892].

Iconografia: Wilson, 1905, loc. cit. (sub L. myriophyllum); Wilson, 1913, loc. cit.; Liang \& Tamura, 2000: fig. 139: 5-6.

Hierbas perennes, de 0,5-2 $\mathrm{m}$ alt. Bulbos anchamente ovoides, hasta de $5 \mathrm{~cm}$ diám.; con escamas carnosas imbricadas de 4-5 × 1-1,5 cm, blancas o a veces amarillentas, sin túnica. Tallos erectos, verde grisáceos, con manchas rojizopurpúreas. Hojas sésiles, alternas, esparcidas, ausentes hacia la base del tallo, sin bulbilos axilares, láminas angostamente lineares, de 5-8 $(-13) \times$ hasta de $1 \mathrm{~cm}$, agudas, 1-nervias, con pocas papilas en márgenes y nervio medio en el envés. Flores horizontalmente expandidas, muy fragantes, terminales, solitarias o en inflorescencias racemosas; pedicelos de 1,8-13 $\mathrm{cm}$ long. Perianto infundibuliforme, tépalos 6, de 9-12 (15) cm long., blancos, a menudo amarillos hacia la garganta y con marcas rosado-purpúreas por fuera, recurvados en el ápice, con nectarios no papilosos ni fimbriados en la base; tépalos externos lanceolados de 1,5-2 cm lat., los internos obovados, hasta de $3,7 \mathrm{~cm}$ lat. Estambres 6, libres, opuestos a los tépalos, incluidos; filamentos esparcidamente papilosos; anteras elipsoides de 0,9-1,2 cm long., amarillas. Ovario súpero, cilíndrico, ca. $2,2 \times 0,3 \mathrm{~cm}$; estilo ca. $6 \mathrm{~cm}$ long.; estigma 3-lobado. Fruto cápsula 3-valva, loculicida; semillas discoides, angostamente aladas, dispuestas como una pila de monedas en cada valva, castañas. $2 n=24$ (Liang \& Tamura, 2000; Tropicos, 2018).

Distribución. Especie endémica de la provincia de Sichuan, China, donde crece entre los 800-2500 m s.n.m., en márgenes de cursos de agua y laderas rocosas, en un área reducida de unos $170 \mathrm{~km}^{2}$ en el valle del río Min, sujeta a los cambios en el uso de las tierras, deslizamientos y terremotos (Matthews, 1986; Liang \& Tamura, 2000; $\mathrm{Wu}$ et al., 2015). Fue introducida a principios del siglo XX en Inglaterra y en los Estados Unidos por el explorador y botánico británico Ernest Henry Wilson (1876-1930), como bulbosa ornamental de vigoroso crecimiento y 
resistencia a las enfermedades. En la actualidad, se encuentra escapada de cultivo en las Islas Británicas, naturalizada y a veces invasora en cultivos en los Estados Unidos y centro-sur de Brasil (Schneider, 2007; Randall, 2017; REFLORA, 2018). Su presencia en cultivo en la Argentina fue indicada por Dimitri (1987). En la provincia de Misiones crece espontánea en bordes de caminos y capueras, en planaltos húmedos, alterados, a 800 m s.n.m., en suelos negros, fértiles, húmedos; comparte el hábitat con la vegetación rupícola en afloramientos basálticos y con gramíneas ruderales en praderas antropizadas. Para nuestro país, es la segunda especie citada de la familia Liliaceae stricto sensu (Tamura, 1998; APG III, 2009; Stevens, 2017), luego de L. longiflorum Thunb. (Hurrell et al., 2010).

Los registros de L. regale para Brasil austral (Paraná, Santa Catarina, Rio Grande do Sul) y el noreste de Argentina (Misiones), tanto los aquí presentados como los incluidos en REFLORA (2018), se enmarcan en el área del Bosque Atlántico del Alto Paraná, por lo cual los datos sobre la naturalización de esta especie aportan a la comprensión de la dinámica de la biodiversidad de esta región ecológica, tanto en sus aspectos florísticos como etnobiológicos, amenazada mayormente por la desforestación (Di Bitetti et al., 2003; Galindo-Leal \& Camara, 2003; Zamudio \& Hilgert, 2012).

Fenología, polinización y dispersión. Florece desde la primavera hasta el otoño, con mayor abundancia durante el verano. Según Ying et al. (2014), la fragancia de las flores atrae insectos polinizadores, y sus compuestos volátiles presentan un patrón de emisión máximo por la noche. Según Wu et al. (2015) la polinización es anemófila y las plantas cultivadas ex situ presentan autopolinización. Las plantas se propagan por semillas, dispersadas por el viento, y también por vía vegetativa a través de sus bulbos.

Nombres vernáculos: azucena, azucena real, lirio-azucena, lirio del rey, lirio real, lirio trompeta (Argentina), lírio-regalo (Brasil).

Usos. Ornamental, por sus flores estivales vistosas y fragantes, se cultiva en suelos fértiles, bien drenados, en sitios a pleno sol; se multiplica fácilmente por bulbos
(Dimitri, 1987; Ellis, 2001; Bradley et al., 2008; Hawthorn, 2009). Se ha ensayado su multiplicación in vitro (Saifullah et al., 2010). Presenta híbridos y cultivares como Lilium regale cv. 'Album', con tépalos blancos sin marcas y anteras anaranjadas, muy difundido (Leendertz, 2014).

La esencia de las flores se utiliza en aromaterapia. Los bulbos, muy amargos, contienen glucósidos (Mimaki \& Sashida, 1990), saponinas esteroides (Mimaki et al., 1993) y compuestos fenólicos con efecto antioxidante (Jin et al., 2012).

Las plantas naturalizadas de $L$. regale y $L$. longiflorum pueden diferenciarse mediante los siguientes caracteres.

\section{Clave para la identificación de las especies naturalizadas de Lilium en Argentina}

1. Bulbos globosos a subglobosos, hasta de $6,5 \mathrm{~cm}$ diám. Hojas linear-elípticas, glabras, a veces con bulbilos axilares. Tépalos blancos, con marcas verdosas por fuera. Filamentos estaminales glabros. L. longiflorum

1'. Bulbos anchamente ovoides, hasta de $5 \mathrm{~cm}$ diám. Hojas angostamente lineares, papilosas en márgenes y nervio medio del envés, sin bulbilos axilares. Tépalos blancos, con marcas rosadopurpúreas por fuera. Filamentos estaminales esparcidamente papilosos. L. regale

Material examinado: ARGENTINA. Misiones: Dep. Gral. Manuel Belgrano, Campiñas de América, camino de tierra al norte de la ruta prov. 17, borde de capuera, 3-IV-2007, Keller et al. 4115 (CTES). BRASIL. Paraná: Município Irati, subespontânea ao longo de rodovias, 4-III-1982, Kummrow 1859 (CTES). Santa Catarina: Município Maravilha: borde de ruta, a $6 \mathrm{~km}$ de Maravilha, 10-XI-1994, Krapovickas \& Cristóbal 44848 (CTES).

\section{Discusión y Conclusiones}

En la Argentina, $L$. regale crece en ambientes perturbados del Bosque Atlántico, asociados a los márgenes de vegetación espontánea alterada (capueras), y a los bordes de caminos y rutas que ofician de "corredores" 
que posibilitan la propagación de especies introducidas (Trombulak \& Frissell, 2000; Birkeland Salvesen, 2006). Los registros para Brasil austral corresponden a los mismos tipos de ambientes, y seguramente presentan un mecanismo de expansión similar. En diversos sitios, los acarreos de tierra y desechos de jardines posibilitan el transporte involuntario de bulbos, de modo semejante al mecanismo de propagación de L. longiflorun (Hurrell et al., 2010) y de otras especies con bulbos u otros órganos subterráneos (Hurrell \& Delucchi, 2007; Hurrell et al., 2009).

En la actualidad, los estudios sobre la biología de las invasiones apuntan a considerar el proceso de la naturalización como un continuum, que puede terminar en una invasión, o no; sin embargo, desde el punto de vista de la naturalización, todas las especies naturalizadas resultan invasoras potenciales. Además, la naturalización varía de acuerdo a las condiciones locales y las capacidades de expansión, reproductiva y/o vegetativa, de diferentes tipos biológicos: leñosas, trepadoras, herbáceas perennes, entre otras (Pyšek \& Richardson, 2006; Richardson et al., 2000, 2010, 2011; Richardson \& Pyšek, 2012). En este contexto, L. regale se considera una especie naturalizada: se ha establecido e integrado a la vegetación local, expandiéndose sin intervención humana directa por medio de sus propios mecanismos de propagación, por vía reproductiva (dispersión anemócora), y mayormente por vía vegetativa.

\section{Agradecimientos}

Los autores expresan su gratitud al personal del Herbario CTES, Instituto de Botánica del Nordeste (IBONE, CONICET), y a los revisores anónimos, cuyos comentarios han enriquecido esta contribución.

\section{Bibliografía}

APG III. 2009. An update of the Angiosperm Phylogeny Group classification for the orders and families of flowering plants: APG III. Bot. J. Linnean Soc. 161: 105-121.

BELL, C., C. WILEN \& A. STANTON. 2003. Invasive plants of horticultural origin. Hortscience 38: 14-16.
BIRKELAND SALVESEN, A. 2006. Escape of introduced ornamentals in Asteraceae. Thesis, University of Oslo, Oslo.

BRADLEY, F. M., B. W. ELLIS \& E. PHILLIPS. 2008. Rodale's Ultimate Encyclopedia of Organic Gardening. Rodale Press, New York.

DEHNEN-SCHMUTZ, K., J. TOUZA, C. PERRINGS \& M. WILLIAMSON. 2007. The horticultural trade and ornamental plant invasions in Britain. Conserv. Biol. 21: 224-231.

DI BITETTI, M. S., G. PlACCI \& L. A. DIETZ. 2003. A Biodiversity Vision for the Upper Parana Atlantic Forest Eco-region: Designing a Biodiversity Conservation Landscape and Setting Priorities for Conservation Action. WWF, Washington DC.

DIMITRI, M. J. 1987. Liliáceas. Encicl. Argent. Agric. Jard. 1 (1): 224-245. Acme, Buenos Aires.

ELLIS, B. W. 2001. Taylor's Guide to Bulbs. Houghton Mifflin Co., New York.

FOXCROFT, L. C., D. M. RICHARDSON \& J. R. WILSON. 2008. Ornamental plants as invasive aliens: problems and solutions in Kruger National Park, South Africa. Environ. Manag. 41: 32-51.

GALINDO-LEAL, C. \& I. G. CAMARA. 2003. Atlantic Forest hotspot status: An overview. En GALINDOLEAL, C. \& I. G. CAMARA (eds.), The Atlantic Forest of South América: Biodiversity Status, Threats, and Outlook, pp. 3-11. Island Press, Washington DC.

GROVES, R., R. BODEN \& M. LONSDALE. 2005. Jumping the Garden Fence: Invasive Garden Plants in Australia. CSIRO-WWF-Australia, Sydney.

HAWTHORN, L. 2009. Gardening with Shape, Line and Texture. Timber Press, Portland.

HURRELL, J. A. \& G. DELUCCHI. 2007. Agapanthaceae, Anthericaeae y Hyacinthaceae (Monocotyledoneae-Asparagales) adventicias en la Argentina. Revista Mus. Argent. Cienc. Nat. (n. s.) 9: 103-107.

HURRELL, J. A., G. DELUCCHI \& F. BUET COSTANTINO. 2009. Nuevas citas de monocotiledóneas adventicias para la Argentina. Revista Mus. Argent. Cienc. Nat. (n. s.) 11: 131-144.

HURRELL, J. A., G. DELUCCHI \& J. A. TOLABA. 2010. Presencia de Lilium longiflorum (Liliaceae) adventicia en la Argentina. Bol. Soc. Argent. Bot. 45: 195-200.

JIN, L., Y. ZHANG, L. YAN, Y. GUO \& L. NIU. 2012. Phenolic compounds and antioxidant activity of bulb extracts of six Lilium species native to China. Molecules 17: 9361-9378.

JSTOR. 2018. Global Plants. Disponible: https:/www. jstor.org/ (Consulta: 27-XI-2018).

KOWARIK, I. 2005. Urban ornamentals escaped from cultivation. En GRESSEL, J. (ed.), Crop ferality and volunteerism, pp. 97-121. CRC Press, Boca Raton.

LEENDERTZ, L. 2014. The Twilight Garden. Pavilion Books, London. 
LIANG, S. \& M. TAMURA. 2000. Lilium. En WU, Z. Y. \& P. H. RAVEN (eds.), Flora of China 24: 135149. Sci. Press, Beijing; Missouri Botanical Garden Press, St. Louis.

MATTHEWS, V. A. 1986. Lilium. En WALTERS, S. M. (ed.), The European Garden Flora 2: 193-206. Cambridge University Press, Cambridge.

MIMAKI, Y. \& Y. SASHIDA. 1990. Novel phenolic amide derivative from the bulbs of Lilium regale. Chem. Pharm. Bull. 38: 541-543.

MIMAKI, Y., Y. SASHIDA, O. NAKAMURA, T. NIKAIDO \& T. OHMOTO. 1993. Steroidal saponins from the bulbs of Lilium regale and $L$. henryi. Phytochemistry 33: 675-682.

PYŠEK, P. \& D. M. RICHARDSON. 2006. The biogeography of naturalization in alien plants. J. Biogeogr. 12: 2040-2050.

RANDALL, R. P. 2017. A Global Compendium of Weeds. Ed. 3. R. P. Randall, Perth.

REFLORA. 2018. Herbário Virtual. Disponible: http:// reflora.jbrj.gov.br/reflora/herbarioVirtual (Consulta: 27-XI-2018).

REICHARD, S. H. \& P. WHITE. 2001. Horticulture as a pathway of invasive plant introductions in the United States. Bioscience 51: 103-113.

RICHARDSON, D. M. \& P. PYŠEK. 2012. Naturalization of introduced plants: ecological drivers of biogeographical patterns. New Phytol. 196: 383-396.

RICHARDSON, D. M., P. PYŠEK, M. REJMÁNEK, M. G. BARBOUR, F. DANE PANETTA \& C. J. WEST. 2000. Naturalization and invasion of alien plants: concepts and definitions. Divers. Distrib. 6: 93-107.

RICHARDSON, D. M., C. C. DAEHLER, M. R. LEISHMAN, A. PAUCHARD \& P. PYŠEK. 2010. Plant invasions: theoretical and practical challenges. Biol. Invasions 12: 3907-3911.

RICHARDSON, D. M., P. PYŠEK \& J. T. CARLTON. 2011. A compendium of essential concepts and terminology in invasion ecology. En RICHARDSON, R. M. (ed.), Fifty Years of Invasion Ecology: The
Legacy of Charles Elton, pp. 409-420. WileyBlackwell, Oxford.

SAIFULLAH, K., N. SHEEBA, R. MARIAN \& K. NAHEED. 2010. Cultivation of lilies (Lilium regale) for commercialization in Pakistan. Pak. J. Bot. 42: 1103-1113.

SCHNEIDER, A. A. 2007. A Flora naturalizada no Estado do Rio Grande do Sul, Brasil: herbáceas subespontâneas. Biociências (Porto Alegre) 15: 257-268.

STEVENS, P. F. 2017. Angiosperm Phylogeny Website. Version 14, July 2017. Disponible: www.mobot.org/ MOBOT/research/APweb (Consulta: 11-IX-2018).

TAMURA, M. 1998. Liliaceae. En KUBITZKI, K. (ed.), The families and genera of vascular plants. III: 343353. Springer, Berlin.

THE PLANT LIST. 2013. The Plant List. Version 1.1. Royal Botanic Gardens, Kew - Missouri Botanical Garden. Disponible: http://www.theplantlist.org (Consulta: 11-IX-2018).

TROMBULAK, S. \& C. FRISSELL. 2000. Review of ecological effects of roads on terrestrial and aquatic communities. Conserv. Biol. 14: 18-30.

TROPICOS. 2018. Tropicos.org. Missouri Botanical Garden. Disponible: http://www.tropicos.org. (Consulta: 11-IX-2018).

WU, Z. H., J. SHI, M. L. XI, F. X. JIANG, M. W. DENG \& S. DAYANANDAN. 2015. Inter-simple sequence repeat data reveals high genetic diversity in wild populations of the narrowly distributed endemic Lilium regale in the Minjiang River Valley of China. PLOS ONE 10 (3): e0118831. https://doi. org/10.1371/journal.pone.0118831.

YING, K., J. R. BAI, X. D. KONG, X. Y. DOU, N. Y. WANG. 2014. Floral scent composition of Lilium regale E. H. Wilson. Acta Hort. 1027: 81-86.

ZAMUDIO, F. \& N. I. HILGERT. 2012. ¿Cómo los conocimientos locales aportan información sobre la riqueza de especies de abejas sin aguijón (Apidae: Meliponini) del Norte de Misiones, Argentina? Interciencia 37: 36-43.

Original recibido el 6 de noviembre de 2018; aceptado el 30 de noviembre de 2018. 
